\title{
TEM Study of Precipitation Behavior of Diluted Cu-Co Alloys
}

\author{
A.L. Rocha*, G. Solórzano*, D. Smith**, M. McCartney** \\ *Department of Material Science and Metallurgy, PUC-Rio, 22451-840, Brazil \\ ${ }^{* *}$ Center for Solid State Science, Arizona State University, USA
}

The $\mathrm{Cu}-\mathrm{Co}$ system has been investigated as a precipitation-hardening alloy for several years using various experimental techniques, especially TEM [1]. These alloys can develop microstructures which promote new interesting magnetic properties such as giant magneto resistance effect (GMR). It has been reported that there are two different precipitation mechanisms occurring in the decomposition of these alloys: homogeneous and heterogeneous precipitation [1].

The alloy composition we are currently investigating ranges from 1 to 4 at $\%$ Co, with the balance being $\mathrm{Cu}$. The samples were homogenized about 30 minutes in the single phase region, quenched and then cold rolled $(70 \%)$ and reanelled in order to promote recrystallization and generated a fine grain size. Isothermal aging treatments were then conducted at temperatures of 500 to $700^{\circ} \mathrm{C}$. Thin specimens for TEM analysis were prepared using the double jet technique in a solution composed, in volume, of $30 \%$ nitric acid and $70 \%$ methanol, kept at $-20^{\circ} \mathrm{C}$.

In the homogeneous mode of decomposition, it is observed in all studied conditions, the presence of Co precipitates which are spherical and coherent with the matrix. These precipitates of about $20 \mathrm{~nm}$ in size show a typical strain field contrast due to elastic distortions of the matrix around the particles, Fig. 1. Hardening is due to the precipitation of these coherent spherical particles. The coexistence of nano-scale coherent precipitates together with micro-scale incoherent precipitates was observed to occur at higher temperatures; the latter forming well developed faceted Co crystals, incoherent with the matrix and ranging from 0.1 to 0.6 in size as shown in Fig. 2.

Discontinuous precipitation (DP) occurred at some grain boundaries under conditions of aging at significant undercooling below the solubility limit and for short times. The samples presenting DP show rod-like particles with regular inter-rod spacing. In general, the rods are not perfect, having a break down along its length. The rare presence of true rods is due to shape instability, especially at high temperatures, where volume diffusion dominates the process [2]. An interesting feature of DP product observed in this system is related to a coagulation process, in which a rod transforms into a columnar array of spherical coherent particles. These spheres are thought to be formed as a consequence of Rayleigh instability [2]. Also, the mobility of the grain boundary is low and attributed to the presence of general coherent particles which form ahead of the reaction front. Analytical TEM has been applied to detect the nature of these particles. EDS line profiles across a particle, stopping the probe at $6 \mathrm{~nm}$ intervals, corroborate that are Co-rich metastable precipitates, as shown in Fig. 3. Typical nanostructural configuration of this system results from a combination of DP of rods driven by migration boundaries together with conventional heterogeneous precipitation at the same boundary when this has seased to migrate, leaving behind an alignment of rods (when it becomes a stationary boundary) and is presented in Fig. 4. The magnetic behavior of these nanostructures is currently under investigation by electron holography.

[1] V. A. Philiphs, Trans. MS AIME 230, 967 (1964).

[2] A. Perovic and G. R. Purdy. Acta Metallurgica Vol. 29, pp. 53-63, 1980. 

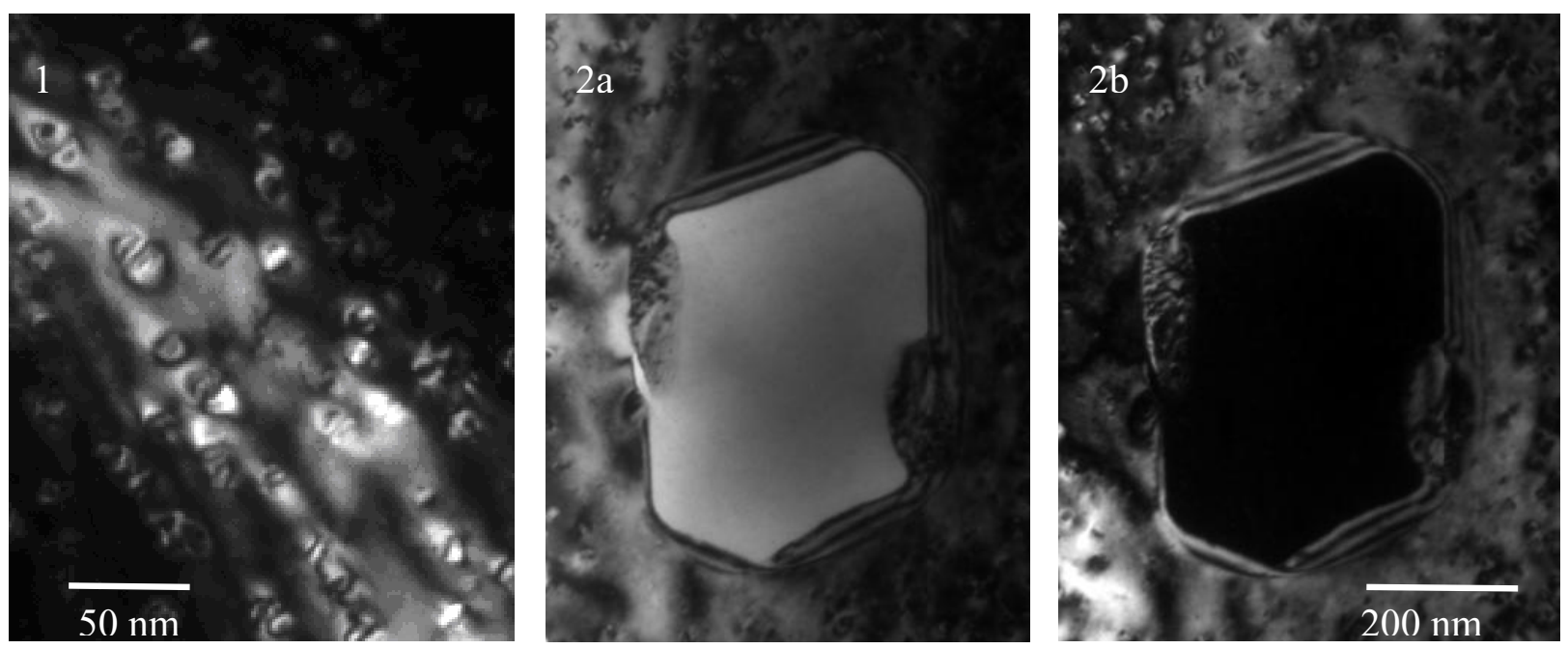

Fig. 1. Centered dark field of coherent nano precipitates.

Fig. 2. (a)Bright Field/(b)Dark Field pair of micrographs showing an incoherent faceted crystal.
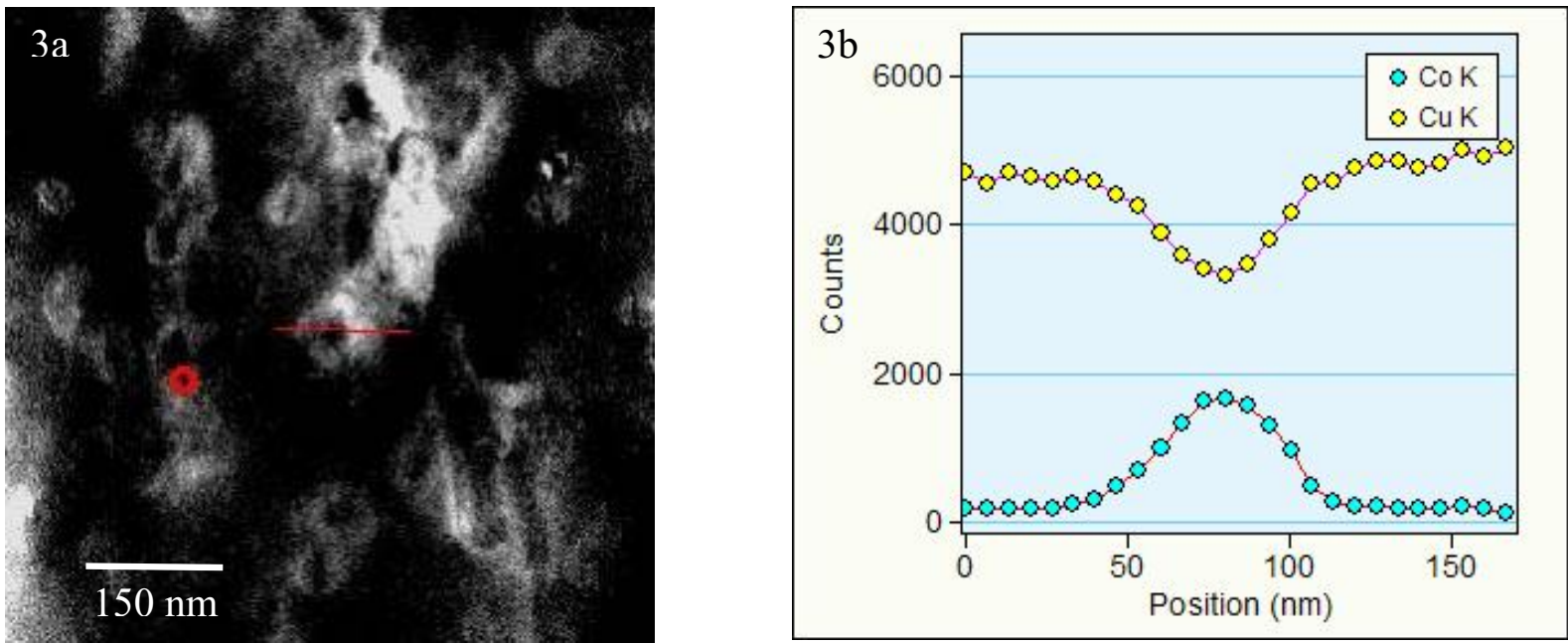

Fig.3. (a) Dark field micrograph of an alloy aged at $500^{\circ} \mathrm{C}$ and (b) the concentration profile across the marked particle.
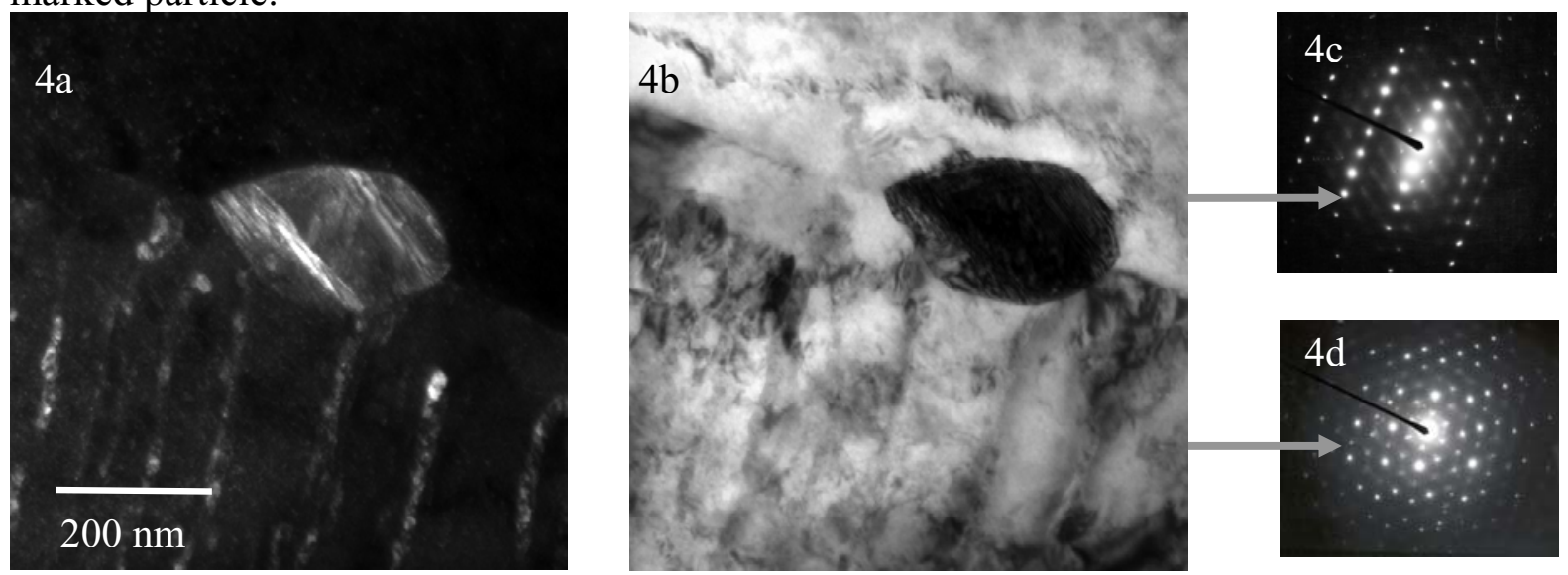

Fig.4- (a) Dark field and (b) bright field image showing DP products and selected area diffraction pattern from parent (c) and product (d) grains. 